\title{
The Heavens in August
}

\author{
A Study of Short Period Variables
}

\author{
By Prof. Henry Norris Russell, Ph.D.
}

THE warm clear nights of summer offer the amateur the best chance for star-gazing in all the year, and, fortunately, he has one of the finest portions of the heavens at his command in the splendid region of the Milky Way, which stretches from Cassiopeia and Cygnus through Aquila to Sagittarius and Scorpio, and forms a vast circle right across the summit of the vault of heaven.

The veriest novice can learn in an hour to identify the principal sky-marks within this region-the zig-zag line of Cassiopeia, now low in the northwest; the great cross of Cygnus, right overhead; the brilliant Altair in Aquila, with a fainter supporting star on each side; the little inverted "Milk Dipper" in Sagittarius, and the long curving line of the tail of Scorpio, dipping close to the horizon, and rising again to a group of brilliant stars at its end.

The possessor of a small telescope will find rich hunting ground here, especially in the region between Aquila and Scorpio, and the student who has only his own eyes to aid him need not lack for interest. For example, there are now easily visible in this region of the heavens four of the most remarkable variable stars in the whole sky, whose changes in light may be readily followed without telescopic aid.

All four belong to the interesting and very puzzling class of objects known as the short-period variables, or, as they are often now called, the Cepheid variables, from their most prominent example, the star Delta Cephei.

This star is shown upon our map, though not marked with its Greek letter, as a near neighbor of $\zeta$ Cephei, which is so marked. Or it may be found by sweeping the eye along the Milky Way from Cassiopeia toward Cygnus. Just about half way between these constellations, and in the very middle of the galaxy, is a little triangle of stars of the fourth magnitude, which is the most prominent configuration in the vicinity. The sides of this triangle are very unequal, and its sharpest angle points toward Cassiopeia. The star at this angle is the variable which we are seeking. Its two neighbors, Zeta and Epsilon Cephei, neither of which is variable, form admirable standards of comparison in estimating its brightness. A few nights of watching, recording each time the brightness of Delta compared with the neighboring stars, will reveal a conspicuous variability. At maximum the star is of magnitude 3.7 , very nearly equal to the brighter of its neighbors, Zeta Cephei, while at minimum it is of magnitude 4.6, which means that it is just about half as bright. A longer study would reveal that the variations in brightness were periodic, the maximum being reached at intervals of about five and one third days. Many years of observation have shown that this periodic variation is perfectly regular. Discovered by Goodricke in $\mathbf{1 7 8 4}$ it has ever since conformed accurately to a period of 5 days 8 hours 47 minutes and 37 seconds, and there is every reason to suppose that it will continue indefinitely to do so. The character of the variation is also always the same. Starting at minimum we will find it rising to maximum in a day and a half and falling in brightness more gradually, so that it takes nearly four days to return to its minimum light, and the changes as to return to its minimum light, and the changes as
observed at different times follow the same curve very observed
closely.

The star Eta $(\eta)$ Aquilae, which is now also easily observable, was found to be variable by Pigott in the same year as that on which Goodricke detected the variations of $\delta$ Cephei, and behaves very similarly, running through almost exactly the same range of visual brightness, but with a period of 7.17638 days. Here again the increase of light is much more rapid than the decrease, occupying some $2 \frac{1 / 4}{4}$ days, or a little less than half as long as the latter.

This star, though not shown on our map, may be very easily found on a line between $\delta$ and $\theta$ Aquilae, and a little nearer the latter. It is nearly as bright as these two stars when at maximum, but much fainter at minimum. The other two variables of this sort which are now easily observable lie farther south and are known as $\omega$ and $\chi$ Sagittarii. The first of these is about a degree north of the bright star $\gamma$ Sagittarii, which is marked on our map, and the second lies about two fifths of the way from this to $\theta$ Ophiuchi (also shown on the map) and is the only bright star near this line. The character of the variation is in both cases very similar to that of the stars previously described. $\omega$ Sagittarii varies from magnitude 4.3 to 5.1 in a period of 7.595 days, the rise in brightness takin about half as long as the fall, and the maximum bein a little more than twice the minimum light. In th case of $\chi$ Sagittarii, the range of variation from magnitude 4.4 to 5.0 makes the minimum brightness 55 pe cent of the maximum, while the period is 7.0118 days and the increase of light (unlike the stars previousl described) is hardly more rapid than the decrease.

These four stars, so similar in their general characteristics, are typical examples of a considerable group of variables, of which between 150 and 200 are now known, though the periods of only about 100 have bee accurately determined. These periods range from about forty days down to three hours-the shortest period of any known variable star. In addition, there are hun-

and but 18 minutes of arc apart, while Neptune is only degree away on the other side. This simultaneous conjunction of three planets is rather remarkable, but as they rise less than an hour earlier than the Sun, Neptune will be utterly invisible, though the other two planets may easily be seen with a telescope (provided with suitable finding circles) even in broad daylight, and in the same low-power field.

Mars, too, is a morning star, rising about 1 A. M. in the middle of the month. He is near the border of Taurus and Gemini and appears about as bright as Castor or Pollux.

Jupiter is in Pisces, approaching opposition, and rises about 9:15 P. M. on the 1st and 7:20 P. M. on the 31st. $\mathrm{He}$ is far brighter than any other celestial object in sight, and is correspondingly conspicuous.

Saturn is a morning star, in the middle of Gemini, and rises about an hour earlier than Mars. Since his rings are opened widely and send us nearly half as much light again as the ball of the planet, he appears much brighter than he did eight years ago, when his rings were edgewise toward us, and nearly equals Capella in brilliancy. Uranus is in opposition on the 7th, and is visible all night long.

There is an unusually good opportunity for identifying him this year, for at the end of the month he passes close to the fourth magnitude star $\theta$ Capricorni. This star, though not shown on our map, may be easily identified, as it lies very nearly half-way between $\beta$ and $\delta$ Capricorni, and a little south of the line joining them.

At the beginning of August Uranus is a little over 1 degree east of this star and about 12 minutes north of it. On September 3rd he passes due south of the star at a distance of 7 minutes, and by the end of September he is some 45 minutes west and 20 minutes south of it. Uranus appears like a star of magnitude 5.7, and about five times fainter than the star which serves as a guide to his position. Early in August and late in September he can be seen with the unaided eye on a clear dark night. Near September 1st he will be too near Theta Capricorni to be observable without an opera glass; but the smallest instrumental aid will make it easy to follow his motion.

Neptune, as already stated, is close to the Sun and invisible this month.

The Moon is in her last quarter at $4: 27$ P. M. on the 2 nd, new at $5: 52$ P. M. on the 10th, in her first quarter at $9: 17$ P. $M$ on the 17th, and new at $4: 40 \mathrm{P}$. M. on the 24th. She is nearest the Earth on the 20th, and farthest away on the 4th. During the month she is in conjunction with

NIGHT SKY: AUGUST AND SEPTEMBER

dreds more of variable stars in the great globular star clusters whose variation is of this type-the periods of these "cluster variables" being usually not far from twelve hours, and many more again in those remarkable isolated outlines of the Milky Way known as the Magellanic clouds, with periods ranging from a couple of days to several months. It is, therefore, evident that in this sort of stellar variation we meet with a very important cosmical phenomenon. To unravel its nature has proved one of the most perplexing problems of astrophysics, and, after many years of effort, a satis factory theory seems still remote. Many interestin facts, at least, have been brought to light; but we must postpene an account of them until next month.

\section{The Heavens.}

The remaining constellations are easily identified Capricornus and Aquarius occupy a dull region in the southeast. The bright star below them is Fomalhaut, in the Southern Fish. Due east we see the great square of Pegasus, and to the left of this Andromeda with Aries below and Perseus farther to the left. The two Bears and Draco are conspicuous in the northwest and north, Lyra is almost overhead, and Hercule Corona and Boötes are below, to the westward, while Ophiuchus and Serpens occupy the southwestern sky.

\section{The Planets.}

Mercury is in conjunction with the Sun, and behind him, on the 14th, and is, therefore; practically invisible all through the month. Venus, too, is almost behind the Sun, and likewise practically invisible. On the morning of the 4th the two planets are in conjunction ing the month she is in conjunction with Mars on the 6th, Saturn on the 7th, Neptune and Venus
on the 9th, Mercury on the 10th, Uranus on the 23rd, and Jupiter on the 26th

At the new Moon on August 10th there is an annular eclipse of the Sun. Though this begins before the Sun has set over the United States, the Moon's shadow strikes the Earth far to the southward, and no eclipse is visible at all from any part of North America.

Observers in Hawaii, however, will see a very large eclipse at noon, with the Sun nearly overhead, and those in the Philippines may observe the end of a partial eclipse just after sunrise. The track along which an annular eclipse is visible extends for a great distance across the South Pacific, but does not pass over any land of importance. The computed duration of this phase is a little over one and a half minutes. Southport, Conn.

\section{How Old Are Oysters?}

$A$ S the result of examinations of the surface lamella $\mathbf{A}_{\text {of the shells of over six hundred oysters of known }}$ age, and of various ages from eighteen months to six years, made by Miss A. L. Massy, and noted in the Scientific Investigations (English) under the section on fisheries, the following conditions prevailed as to the relation between age and the number of surface lamellæ, or growth rings. Miss Massy states that an oyster of eighteen months or two summers appears to possess at least two rings, but may have as many as five; one of three summers has at least two rings, but may have six. A four-year-old oyster may have only three rings, or may possess seven or eight. 